\title{
Crosstalk analysis of dysregulated pathways in preeclampsia
}

\author{
TAO WANG ${ }^{1}$, XING-ZHEN SHI $^{2}$ and WEN-HUA WU ${ }^{3}$ \\ ${ }^{1}$ Department of Obstetrics, Chengdu Women and Children Center Hospital, Chengdu, \\ Sichuan 610000; ${ }^{2}$ Department of Gynaecology and Obstetrics, First People's Hospital of Jinan, \\ Jinan, Shandong 250011; ${ }^{3}$ Department of Orthopedics, The Second Affiliated Hospital \\ of Fujian Medical University, Quanzhou, Fujian 362000, P.R. China
}

Received June 26, 2018; Accepted January 9, 2019

DOI: $10.3892 / \mathrm{etm} .2019 .7178$

\begin{abstract}
A crosstalk between multiple biological pathways has been proposed in biological processes. However, the existence and degree of this phenomenon in patients with preeclampsia (PE) have not been strictly investigated. Thus, this study explored an dysregulated pathway set (DPS) for PE based on pathway crosstalk network $(\mathrm{PCN})$ related analysis. In the present study, four steps were performed in the inference of DPS: acquiring data of gene expression, pathway and protein-protein interaction (PPI) construction; building a PCN through integrating the information in these datasets and Pearson's correlation coefficient (PCC). A principal component analysis (PCA) approach was used to compute the activity of every pathway for selecting seed pathway of PCN. DPS was evaluated by measuring of an area under the receiver operating characteristics curve (AUC) and seed pathway from PCN. Consequently, a total of 420 pathways and 6,032 crosstalks were mapped to the PCN, in which RIG-I/MDA5-mediated induction of IFN- $\alpha / \beta$ pathways was identified as the seed pathway that had the greatest changes in activity scores across PE patients and normal controls. DPS was composed of 15 dysregulated pathways and 46 crosstalks, in which CLEC7A (Dectin-1) signaling possessed the highest degree of 12 , which indicated it exerted an important role in the DPS. Our results revealed crosstalk between pathways and the DPS crucial for PE pathogenesis, which aid in excavating potential biomarkers of PE therapy and unveil the underlying pathological mechanism of this disease.
\end{abstract}

\section{Introduction}

Preeclampsia (PE) is a pregnancy-induced hypertension affecting 3-5\% of nulliparous pregnancies (1). The risk of

Correspondence to: Dr Wen-Hua Wu, Department of Orthopedics, The Second Affiliated Hospital of Fujian Medical University, 34 Zhongshan Northern Road, Quanzhou, Fujian 362000, P.R. China E-mail:wenhuawuqz@163.com

Key words: preeclampsia, data, pathway crosstalk network, dysregulated pathway set renal dysfunction, red blood cell breakdown, and impaired liver function is increased due to the occurrence of PE (2). For pregnant woman, PE is considered to be the major causes of morbidity and mortality worldwide, and it is reported that approximately 70,000 pregnant women and 500,000 infant die of it every year (3). However, the pathological mechanism underlying $\mathrm{PE}$ is imperfect and inconsistent. There are two authoritative theories, one is the vascular aspects of pathophysiology, such as placental hypoxia, endothelial dysfunction and enhancement of platelet aggregation (4); and the other is immune dysfunction that participates in the onset and duration of the clinical manifestations in fetal tissue $(5,6)$. PE is likely to be involved in genetic, immune and biological combinations that make it a heterogeneous disease. This property increases the difficulty in designing efficient therapies and identifying specific biomarkers as targets for PE patients.

Numerous researchers have made great efforts to uncover prominent biomarkers for targeted treatment of PE samples and reveal pathological mechanism for this disease $(7,8)$. Yong et al revealed that various susceptibility genes, such as angiotensinogen (AGT), interleukin-6 (IL6), and transforming growth factor $\beta 1$ (TGF $\beta 1)$, played an important role in the occurrence and development of PE by affecting the apoptotic ability and transmission of cellular signals (7). However, the results from various studies are usually inconsistent due to the difference of experimental samples, analyzing methods and batch parameters (9). Besides, from a biological perspective, genes function in complex disease through interacting with each other rather than as an independent entity (10). Due to the genes usually functioning in the form of pathways to perform certain biological functions, it is necessary to identify pathways by extracting potential biology from a co-operated large gene list (11). This also provides a novel insight to investigate potential biomarkers for PE.

Similar to a protein-protein interaction (PPI) network where nodes stand for genes and edges are PPIs, pathways and pathway-pathway interaction (crosstalk) between any two pathways could build a pathway crosstalk network (PCN) which integrate the data of pathway and PPI. Examination of pathway alterations or identification of abnormal pathways from a PCN contribute to form a biological information characterization that occurs in a specific dataset, which may help 
the formation of new possibilities and identification of genetic characteristics for understanding, diagnosis and treatment of disease $(12,13)$. Therefore, in the present work, we aimed to identify a dysregulated pathway set (DPS) between PE patients and normal controls based on PCN. The findings might make a contribution to potential biomarkers for PE and reveal the pathological mechanism underlying PE.

\section{Materials and methods}

There were four steps in the inference of DPS: firstly, gene expression data, pathway and PPI were obtained; secondly, collected datasets and Pearson's correlation coefficient (PCC) were integrated to construct a PCN; thirdly, on the basis of the principal component analysis (PCA) method, a seed pathway of PCN was chosen through calculating pathway activity of each pathway; finally, DPS from PCN was extracted by analyzing the area under the receiver operating characteristics curve (AUC) index and the seed pathway.

\section{Datasets}

Gene expression data. Gene expression datasets (E-GEOD-6573 (14) and E-GEOD-48424 (15) for PE patients and normal controls were downloaded from the ArrayExpress database. Specifically, E-GEOD-6573 consisted of 3 PE samples (Decidua Preeclampsia, Placenta Preeclampsia and Fat Preeclampsia) and 3 normal controls (Decidua Control, Placenta Control and Fat Control), deposited on the GPL570 platform of Affymetrix Human Genome U133 Plus 2.0 Array (Affymetrix; Thermo Fisher Scientific, Inc., Waltham, MA, USA). Additionally, microarray data (E-GEOD-48424) with 19 PE specimens, including 6 women with non-severe PE and 13 with severe PE, and 19 normal healthy specimens (no history of medical illness or medication, and received routing prenatal care) were acquired on the basis of the GPL6480 platform (Agilent Technologies, Santa Clara, CA, USA). Finally, $22 \mathrm{PE}$ specimens and 22 healthy controls were employed in this study.

To eliminate batch effects caused by the use of different experimentation plans and methodologies between E-GEOD-6573 and E-GEOD-48424, the data were merged using COMBAT method in inSilicoMerging package (16). Subsequently, in order to control the quality of the merged data on probe level, a standard approach was applied, in which a Robust Multi-array Average (RMA) approach was employed to calculate the background correction (17), normalization was performed with quantiles algorithm (18), perfectly matched and imperfectly matched revisions were conducted using Micro Array Suite (MAS) method (19), and summary of expression values was performed with median polish method (17). Probes without invalid and duplicates were converted into gene symbols through annotation packages (20). Ultimately, 11,269 genes were acquired for further study from gene expression data.

Pathway data. Reactome pathway database, an open resource for manually-planned pathways, provided an infrastructure for calculation across the biological response networks which was chosen to obtain human-predefined biological pathways (21). In consequence, a total of 1,675 pathways were gained. In order to reinforce the associations of these pathways with PE, we only chose the pathways containing the intersections with the gene expression data. In addition, there was not enough biological information for gene-less pathways, but too generic for pathways with too many genes (22). Hence, only pathways of the number of intersecting genes from 5 to 100 were selected for further study. A total of 1,134 pathways were retained eventually, regarded as pathway data for subsequent analysis. Here, for the convenience of description, every pathway in pathway data was assigned to an ID according to its alphabetical order.

PPI data. In this study, PPI data were extracted from Search Tool for the Retrieval of Interacting Genes/Proteins (STRING) database, which gave a crucial evaluation and integration associated with physics and function of PPIs (23). There were 16,730 genes and 787,896 interactions in the STRING database, but a certain number of them were non-effective interactions or weak relationships with others; thus, we abandoned the interactions of inherent score $<0.2$. Interactions that were reserved were intersection with gene expression data to enhance the correlations and confidence with PE, namely, PPI data. Notably, approximately 293,155 interactions among proteins were involved in the PPI dataset in 9,986 distinctive human genes.

PCN construction. As described above, a PCN was built using each node that represented a pathway, in which one edge was placed in two pathways that had a crosstalk (24). The goal of the crosstalk was to meet the interaction for one of the two conditions, it required that at least one gene was shared for the two pathways of a crosstalk. Furthermore, there should be a common gene that was a differentially expressed gene (DEG) between PE specimen and control groups. Particularly, DEGs were identified for confirming whether there are significant or non-significant differences between two group means by Student's t-test (25). On the basis of unpaired two-tailed $\mathrm{t}$-statistics in $\mathrm{PE}$ and control conditions, gene with $\mathrm{P}$-value $<0.05$ was considered as a DEG.

On the other hand, two genes encoding a pair of interacting genes applied in generation of crosstalk between two pathways were highly co-expressed across the PE and normal groups (weight $\geq 0.8$ ). Otherwise, the crosstalk would be discarded. Note that for an interaction of a pair of pathways, we first calculated the PCC value in two groups separately, and then computed the difference of the two PCC values, at last defined the absolute difference of PCC value as the weight for this interaction. PCC is a common measure for the correlation strength of a pair of variables, values between -1 and +1 were chosen (26). The PCC between gene $x$ and $y$ was calculated as:

$$
\operatorname{PCC}(x, y)=\frac{1}{n-1} \sum_{i=1}^{n}\left(\frac{g(x, i)-\bar{g}(x)}{\sigma(x)}\right) \cdot\left(\frac{g(y, i)-\bar{g}(y)}{\sigma(y)}\right)
$$

Where $\mathrm{n}$ represented the number of specimens in the gene expression dataset; $g(x, i)$ or $g(y, i)$ represented the expression value of gene $x$ or $y$ in the specimen $i$ under a specific group; $\bar{g}(x)$ or $\bar{g}(y)$ was the mean expression value of gene $x$ or $y$.

Seed pathway selection. In order to investigate biological functions for each pathway in PCN, the PCA approach was applied in the summary of the activity score that was 
considered as the level of all genes belonging to a pathway (27). Through retaining the variance in the data and converting the data into a low-dimensional level, internal structure in the high-dimensional data set was efficiently represented using the PCA algorithm (24). Briefly, the data were collected in a matrix $X$ with $j$ samples $(j=1,2, \ldots, J)$ and $k$ pathways $(k=1$, $2, \ldots, K)$. Therefore, a single variable of $X$ was expressed by $x_{k}$ and was a vector in the $J$-dimensional space, abbreviated as $x_{j k}$. Activity score $S_{k j}$ of pathway $k$ in sample $j$ was confirmed by computing the linear combination expression of all the genes in a pathway:

$$
S_{k j}=w_{1 j k} x_{1 j k}+w_{2 j k} x_{2 j k} \cdots+w_{i j k} x_{i j k}
$$

Where $x_{i j k}$ was the standardized expression value of gene $i$ from pathway $k$ in sample $j$, and $w_{i j k}$ was weight of $x_{i j k}$. In particular, the first major composition of corresponding PCA was used to evaluate activity score of pathways. Notably, there was a difference in the activity score of one pathway between the disease and healthy control groups, and a correlation to disease might be shown by the difference, a closer correlation between the pathway and disease could be revealed from the bigger difference. Hence, in this work, the maximally altered activity scoring pathway between PE specimens and normal controls was denoted as PE patient's seed pathway.

DPS identification. The difficulty of understanding was increased because of the existence of numerous genes and interactions in a network, the identification of sub-networks from the global network was a good approach for studying one disease. Therefore, we explored a DPS from the PCN to obtain informative biomarkers for PE. The identification of DPS was regarded as a charateristic selectivity question in a machine study frame after the activity score of pathways in PCN was defined, in which the minimal abnormal pathways effectively distinguished the disease from control were deemed more likely to be dysregulated pathways. Support vector machine (SVMs) model, a kernel-based approach used in the samples with high-dimensional variables, was applied to conduct the feature selection in our study (28). From the seed pathway as a starting point, the second pathway integrated with the seed pathway that had better classification results was found in the pathway of PCN interaction with the seed pathway. New pathways were added to confirm biomarkers of pathway by repeating this procedure until no more pathways were selected to increase the accuracy of the classification. AUC index was used to evaluate the prediction accuracy or classification capacity (29), and five-fold cross validation was applied to test the performance ability (30). In the five-fold cross validation, five equal-size parts were randomly syncopated from all specimens, four of which were used as training sets and the last one was selected as test set to examine the classification property. AUC was repeatedly calculated 100 times as the addition of new pathways and the average was applied as the ultimate data.

\section{Results}

$P C N$. As mentioned above, there were 11,269 genes, 1,134 pathways and 293,155 PPIs in the data of gene expression, pathway and PPI, respectively. Using the Student's t-test, 1,593 DEGs
Table I. Top 10 crosstalks in pathway crosstalk network (PCN).

\begin{tabular}{crcc}
\hline \multicolumn{3}{c}{ Crosstalk } \\
\cline { 2 - 3 } Rank & Pathway (ID) & Pathway (ID) & Weight \\
\hline 1 & 74 & 261 & 261.89 \\
2 & 261 & 806 & 260.09 \\
3 & 261 & 787 & 244.15 \\
4 & 74 & 990 & 243.86 \\
5 & 806 & 990 & 233.54 \\
6 & 76 & 261 & 229.18 \\
7 & 787 & 990 & 227.53 \\
8 & 74 & 793 & 227.00 \\
9 & 793 & 806 & 226.36 \\
10 & 261 & 990 & 225.53
\end{tabular}

The pathway name for ID: 74, APC/C (Anaphase-promoting complex)-mediateddegradationofcellcycleproteins;76,APC/C-Cdc20 (Anaphase-promoting complex) - (Cell division cycle 20) mediated degradation of mitotic proteins; 261, DNA Replication; 787, Regulation of APC/C activators between G1/S and early anaphase; 793, Regulation of DNA replication; 806, Regulation of mitotic cell cycle; 990 , Synthesis of DNA.

were detected between PE patients and normal controls from the gene expression data. A crosstalk between two pathways that met one of the same genes of the two pathways was defined as DEG, or co-expression in the two pathways was upregulated, it would be reserved to prepare for the construction of PCN. As a result, 122,946 crosstalks were obtained. To decrease the complexity of large-scale networks, the top 5\% of all crosstalks in descending order of their weight was chosen. Consequently, 6,032 crosstalks among 420 pathways were retained to form the PCN, as illustrated in Fig. 1. Specifically, we listed the top 10 crosstalks in PCN with higher weights than others in Table I. Consequently, we obtained 7 pathways that were enriched in 10 crosstalks, interestingly, DNA replication actively participated in 5 of 10 crosstalks. It showed that the crosstalk between APC/C-induced alteration of cell cycle proteins (ID: 74) and DNA Replication (ID: 261) was the most significant one with the highest weight $=261.89$.

Seed pathway. On the basis of PCA method, the activity score of each pathway was computed to examine the key pathways in PCN. A significant difference was found in the activity scores between PE specimens and normal controls. Consequently, it is a huge challenge how to conquer inconsonant results and establish criteria to evaluate the significance of pathways. After careful consideration, the absolute alteration in activity scores between PE patients and normal controls was expressed as its ultimate activity score. In addition, the activity score of pathway with the largest alteration was regarded as the seed pathway. The result showed that the number of pathways was reduced with the increase of the absolute alteration. Importantly, the induction of IFN- $\alpha / \beta$ pathways mediated by RIG-I/MDA5 was the seed pathway for PE with the absolute alteration of activity score equal to 4.49. 


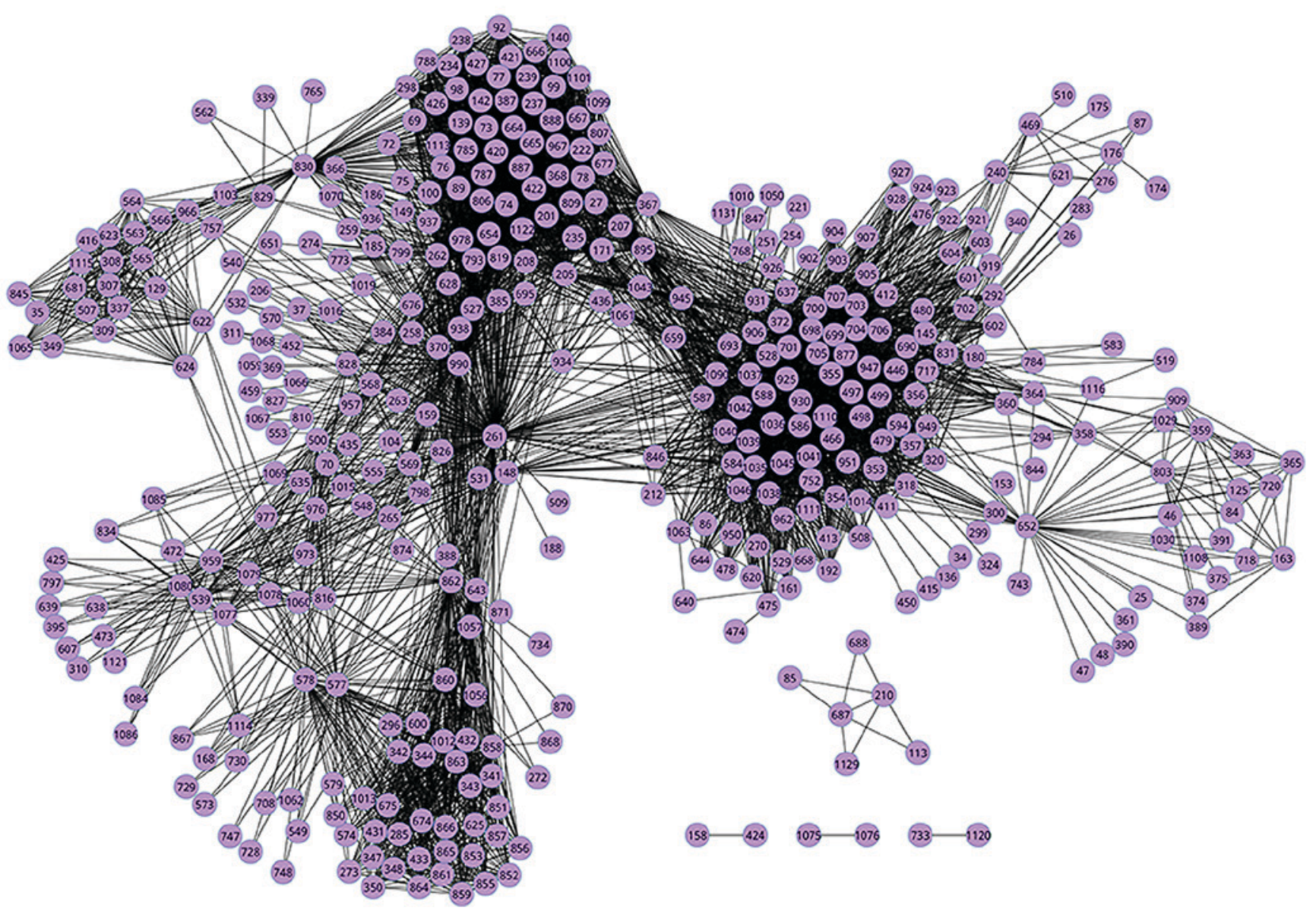

Figure 1. Pathway crosstalk network (PCN) for preeclampsia (PE). There were 1,134 pathways and 6,147 crosstalks in the PCN. Each circle represents a pathway, and the line between any two circles indicates that crosstalk exists between a pair of pathways.

DPS. Based on the seed pathway, pathways were selected to extract DPS from the PCN for improving its AUC, and the process stopped when AUC started to drop. Eventually, DPS (AUC $=0.928)$ was acquired for PE, indicating that DPS had better prediction accuracy and classification property between PE specimens and normal controls. The dysregulated pathways of PE patients were the pathways in the DPS, including the seed pathway RIG-I/MDA5 mediated induction of IFN- $\alpha / \beta$ pathways. Besides, network diagram for DPS is shown in Fig. 2, and 15 pathways and 46 crosstalks were deposited. Furthermore, we computed the topological degree for each node by summing up the number of its adjacent pathways, and the result is described in Table II. We found that the degree for CLEC7A (Dectin-1) signaling (ID: 171) was the highest among the 15 dysregulated pathways. When studying the gene compositions, the seed pathway was composed of 62 genes that contained 12 DEGs between PE patients and normal controls (Table II). Among the 15 dysregulated pathways, CLEC7A (Dectin-1) signaling (ID: 171) had the largest gene size of 89, while Toll Like Receptor TLR1:TLR2 Cascade (ID: 1041) had the most number of DEGs of 21.

\section{Discussion}

Pathway analysis decreases the complexity, enhances the explanatory power and it is a good choice for in-depth underlying biology of genes and proteins (31). Nevertheless,

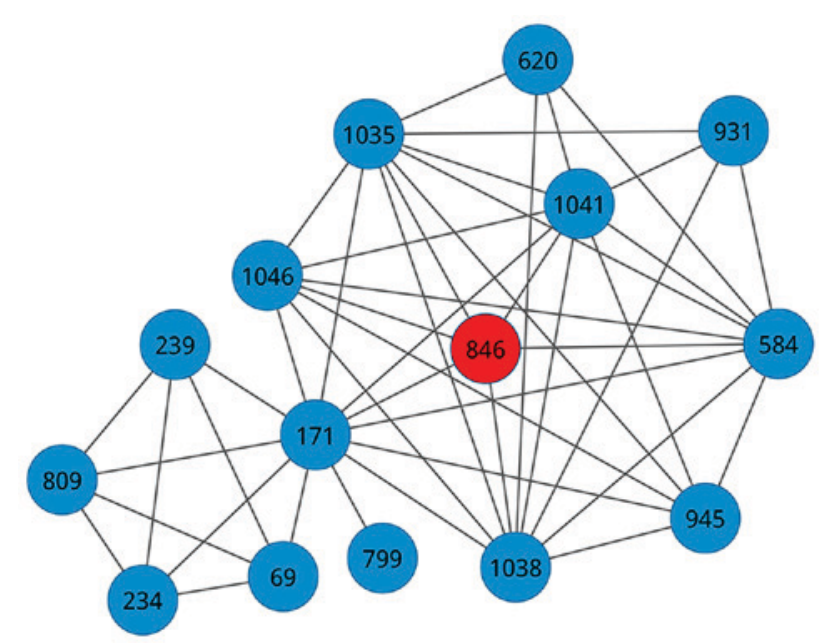

Figure 2. Network diagram for the dysregulated pathway set (DPS) for preeclampsia (PE). Each circle represents a pathway, and the line between any two circles indicate that a crosstalk exists between a pair of pathways. The red node is the seed pathway. The ID verse dysregulated pathway: 69, Antigen processing-Cross presentation; 171, CLEC7A (Dectin-1) signaling; 234, degradation of AXIN; 239, Degradation of GLI2 by the proteasome; 584, MyD88 cascade initiated on plasma membrane; 620, NOD1/2 Signaling Pathway; 799, Regulation of hypoxia-inducible factor (HIF) by oxygen; 809, Regulation of ornithine decarboxylase (ODC); 846, RIG-I/MDA5 mediated induction of IFN- $\alpha / \beta$ pathways; 931 , Signaling by Leptin; 945 , Signaling by TGF- $\beta$ Receptor Complex; 1035, Toll Like Receptor 10 (TLR10) Cascade; 1038, Toll Like Receptor 5 (TLR5) Cascade; 1041, Toll Like Receptor TLR1:TLR2 Cascade; and 1046, TRAF6 Mediated Induction of proinflammatory cytokines. 
Table II. Properties of dysregulated pathways in dysregulated pathway set (DPS).

\begin{tabular}{rlccc}
\hline \multicolumn{1}{r}{ ID } & \multicolumn{1}{c}{ Dysregulated pathway } & $\begin{array}{c}\text { Number of } \\
\text { genes }\end{array}$ & $\begin{array}{c}\text { Number of } \\
\text { DEGs }\end{array}$ & Degree \\
\hline 846 & RIG-I/MDA5 mediated induction of IFN- $\alpha / \beta$ pathways & 62 & 12 & 6 \\
1041 & Toll Like Receptor TLR1:TLR2 Cascade & 85 & 21 & 9 \\
1046 & TRAF6 Mediated Induction of proinflammatory cytokines & 68 & 16 & 7 \\
584 & MyD88 cascade initiated on plasma membrane & 75 & 17 & 9 \\
1035 & Toll Like Receptor 10 (TLR10) Cascade & 75 & 17 & 9 \\
1038 & Toll Like Receptor 5 (TLR5) Cascade & 75 & 17 & 9 \\
171 & CLEC7A (Dectin-1) signaling & 89 & 13 & 6 \\
945 & Signaling by TGF- $\beta$ Receptor Complex & 68 & 12 & 4 \\
234 & Degradation of AXIN & 40 & 7 & 4 \\
809 & Regulation of ornithine decarboxylase (ODC) & 23 & 6 & 4 \\
931 & Signaling by Leptin & 54 & 6 & 4 \\
239 & Degradation of GLI2 by the proteasome & 26 & 3 & 4 \\
620 & NOD1/2 Signaling Pathway & 24 & 12 & 1 \\
799 & Regulation of Hypoxia-inducible Factor (HIF) by oxygen & 73 & & 4 \\
69 & Antigen processing-Cross presentation & & & 4 \\
\hline
\end{tabular}

previous studies mainly focused on discovering disrupted pathways between normal and disease samples or the common genes between two altered pathways (32), ignoring the fact that pathways can influence each other via a crosstalk instead of independent actions (33). Pathway crosstalk can promote the prevention and therapy of certain diseases through inhibiting the interaction between pathways that play an important role in the invasive and proliferative capacities of diseased cells (34). A network-based method can extract information and important genes that are relied on, in the bio-molecular network, not a single gene (35). In addition, it has been utilized to analyze PPI data and deeply understand the mechanisms of biological system operation (36). Herein, PPIs provide valuable information on how genes can fulfil their biological functions, and develop a global interaction network that clarifies the overall organization and interaction among functions that are vital to the survival and growth of cells (11).

To the best of our knowledge, genetic screening is an effective biological method that identifies the interaction of a pathway, in which the synthetic lethality of both mutations usually shows that the two mutations exist with the interaction between the two pathways, respectively (37). This genetic interaction can also be employed to connect closely-packed proteomes in protein networks (38). In the present study, the data of gene expression, pathway and PPI were systematically integrated to construct the PCN of PE using a computational approach. PCA method was used to identify the seed pathway by calculating the activity score of the pathway in PCN. Starting from the seed pathway, the DPS was extracted from PCN based on AUC, and pathways in DPS were defined as dysregulated. Pathways in DPS that were delineated as distinguishing features between PE specimens and normal controls were reasonable and biologically interpretable. Briefly, the first pathway biomarker was the seed pathway that was able to greatly distinguish the disease group and normal group, and through identifying the pathways that interacted with the seed pathways in PCN, the integration of second pathway and the first pathway was performed to obtain better classification results.

A total of 420 pathways and 6,032 crosstalks were mapped to the PCN, in which induction of IFN- $\alpha / \beta$ pathways mediated by RIG-I/MDA5 was identified as seed pathway. Functionally, the interferon (IFN) genes were upregulated by RIG-I and MDA5 in a similar manner, but they respond differently to different virus species (39). RIG-I and MDA5 signaling caused the activation and phosphorylation of two serine/threonine kinases for p-IRF3 and p-IRF7, and IRF3 and IRF7 after phosphorylation translocated to the nucleus and then mediate transcription of IFNA and IFNB genes (40). The association between the pathway and PE was reported by Chatterjee et al (41).

The DPS was composed of 15 dysregulated pathways and 46 crosstalks, in which CLEC7A (Dectin-1) signaling (ID: 171) possessed the highest degree of 12 , which indicated its important role in the DPS. Coincidentally, this pathway comprised of 89 genes which was the largest size among the 15 dysregulated pathways. Obviously, different pathways might affect each other, especially when the DEGs with significant alterations in expression levels overlap (42). Hence, we detected the number of DEGs enriched in the dysregulated pathways of DPS, and the result uncovered that Toll Like Receptor TLR1:TLR2 Cascade (ID: 1041) consisted of the most number of DEGs of 21 .

There are several limitations in this study. Due to the limited number of samples in E-GEOD-6573 and E-GEOD-48424, we did not divide the PE patients according to the severity of PE. Further studies are required on various aspects including, more clinical samples, placental mesenchymal trophoblasts from patients with normal late pregnancy and PE patients, and key protein expression in CLEC7A (dectin-1), signaling in function of these cells need exploring. A more molecular mechanism will be investigated. 
In conclusion, a DPS with 15 dysregulated pathways and 46 crosstalks from the PCN were identified through integrating the data of gene expression, pathway and PPI. Although we analyzed the crosstalk of the pathways and identified the major pathway, a further examination on whether the pathway interactions are worthwhile needs clarification. With high-throughput genomic technology becoming more economical and accurate, the application may become more common for identifying potential target genes.

\section{Acknowledgements}

Not applicable.

\section{Funding}

No funding was received.

\section{Availability of data and materials}

The datasets used and/or analyzed during the current study are available from the corresponding author on reasonable request.

\section{Authors' contributions}

TW performed the experiments and analyzed the data, and was also a major contributor in writing the manuscript. XZS made a substantial contribution to the study conception and design. TW and XZS performed the statistical analysis. WHW was involved in the conception and design of the manuscript and gave the final approval for publication. All authors read and approved the manuscript.

\section{Ethics approval and consent to participate}

Not applicable.

\section{Patient consent for publication}

Not applicable.

\section{Competing interests}

The authors declare that they have no competing interests.

\section{References}

1. Villar J, Say L, Gülmezoglu A, Merialdi M, Lindheimer MD, Betran AP and Piaggio G: Eclampsia and Pre-Eclampsia: A worldwide health problem for 2000 years. In: Pre-Eclampsia Critchley H, Maclean A, Poston L and Walker J (eds). RCOG Press, London, pp189-207, 2003.

2. Al-Jameil N, Aziz Khan F, Fareed Khan M and Tabassum H: A brief overview of preeclampsia. J Clin Med Res 6: 1-7, 2014.

3. Kenny LC, Black MA, Poston L, Taylor R, Myers JE, Baker PN, McCowan LM, Simpson NAB, Dekker GA, Roberts CT, et al: Early pregnancy prediction of preeclampsia in nulliparous women, combining clinical risk and biomarkers: The Screening for Pregnancy Endpoints (SCOPE) international cohort study. Hypertension 64: 644-652, 2014

4. Myatt L, Redman CW, Staff AC, Hansson S, Wilson ML, Laivuori H, Poston L, Roberts JM and Colaboratory GP; Global Pregnancy CoLaboratory: Strategy for standardization of preeclampsia research study design. Hypertension 63: 1293-1301, 2014.
5. Redman CWG and Sargent IL: Immunology of pre-eclampsia. Am J Reprod Immunol 63: 534-543, 2010.

6. Eiland E, Nzerue C and Faulkner M: Preeclampsia 2012. J Pregnancy 2012: 586578, 2012.

7. Yong HE, Melton PE, Johnson MP, Freed KA, Kalionis B, Murthi P, Brennecke SP, Keogh RJ and Moses EK: Genome-wide transcriptome directed pathway analysis of maternal preeclampsia susceptibility genes. PLoS One 10: e0128230, 2015.

8. Long W, Shi Z, Fan S, Liu L, Lu Y, Guo X, Rong C, Cui X and Ding H: Association of maternal KIR and fetal HLA-C genes with the risk of preeclampsia in the Chinese Han population. Placenta 36: 433-437, 2015.

9. Grill S, Rusterholz C, Zanetti-Dällenbach R, Tercanli S, Holzgreve W, Hahn S and Lapaire O: Potential markers of preeclampsia - A review. Reprod Biol Endocrinol 7: 70, 2009.

10. Bi D, Ning H, Liu S, Que X and Ding K: Gene expression patterns combined with network analysis identify hub genes associated with bladder cancer. Comput Biol Chem 56: 71-83, 2015.

11. Li Y, Agarwal P and Rajagopalan D: A global pathway crosstalk network. Bioinformatics 24: 1442-1447, 2008.

12. Doniger SW, Salomonis N, Dahlquist KD, Vranizan K, Lawlor SC and Conklin BR: MAPPFinder: Using Gene Ontology and GenMAPP to create a global gene-expression profile from microarray data. Genome Biol 4: R7, 2003.

13. Dawson JA and Kendziorski C: An empirical Bayesian approach for identifying differential coexpression in high-throughput experiments. Biometrics 68: 455-465, 2012.

14. Herse F, Dechend R, Harsem NK, Wallukat G, Janke J, Qadri F, Hering L, Muller DN, Luft FC and Staff AC: Dysregulation of the circulating and tissue-based renin-angiotensin system in preeclampsia. Hypertension 49: 604-611, 2007.

15. Textoris J, Ivorra D, Ben Amara A, Sabatier F, Ménard JP, Heckenroth H, Bretelle F and Mege JL: Evaluation of current and new biomarkers in severe preeclampsia: A microarray approach reveals the VSIG4 gene as a potential blood biomarker. PLoS One 8: e82638-e82638, 2013.

16. Taminau J, Meganck S and Lazar C: Using the inSilicoMerging package. https://bioc.ism.ac.jp/packages/2.11/bioc/vignettes/ inSilicoMerging/inst/doc/inSilicoMerging.pdf

17. Irizarry RA, Bolstad BM, Collin F, Cope LM, Hobbs B and Speed TP: Summaries of Affymetrix GeneChip probe level data. Nucleic Acids Res 31: e15-e15, 2003.

18. Bolstad BM, Irizarry RA, Astrand M and Speed TP: A comparison of normalization methods for high density oligonucleotide array data based on variance and bias. Bioinformatics 19: 185-193, 2003.

19. Bolstad B: Affy: Built-in processing methods. 2003. https:// users.soe.ucsc.edu/ leslie/MOUSE/chucktest/src/affy/doc/builtinMethods.pdf

20. Allen JD, Wang S, Chen M, Girard L, Minna JD, Xie Y and Xiao G: Probe mapping across multiple microarray platforms. Brief Bioinform 13: 547-54, 2012.

21. Croft D, Mundo AF, Haw R, Milacic M, Weiser J, Wu G, Caudy M, Garapati P, Gillespie M, Kamdar MR, et al: The Reactome pathway knowledgebase. Nucleic Acids Res 42: D472-D477, 2014.

22. Ahn T, Lee E, Huh N and Park T: Personalized identification of altered pathways in cancer using accumulated normal tissue data. Bioinformatics 30: i422-i429, 2014.

23. Szklarczyk D, Franceschini A, Wyder S, Forslund K, Heller D, Huerta-Cepas J, Simonovic M, Roth A, Santos A and Tsafou KP: STRING v10: Protein-protein interaction networks, integrated over the tree of life. Nucleic Acids Res 43: D447-D452, 2015.

24. Liu KQ, Liu ZP, Hao JK, Chen L and Zhao XM: Identifying dysregulated pathways in cancers from pathway interaction networks. BMC Bioinformatics 13: 126, 2012.

25. Haynes W: Student's t-test. In: Encyclopedia of Systems Biology. Dubitzky W, Wolkenhauer O, Cho KH and Yokota H (eds). Springer, New York, NY, 2013.

26. Nahler G: Pearson correlation coefficient. In: Dictionary of Pharmaceutical Medicine. Springer, Vienna, pp132, 2009.

27. Bro R and Smilde AK: Principal component analysis. Anal Methods 6: 2812-2831, 2014.

28. Chang C-C and Lin C-J: Libsvm: A library for support vector machines. ACM Trans Intell Syst Technol 2: 27, 2011.

29. Huang J and Ling CX: Using auc and accuracy in evaluating learning algorithms, knowledge and data engineering. IEEE T Knowl Data En 17: 299-310, 2005. 
30. Rojatkar DV, Chinchkhede KD and Sarate GG: Handwritten Devnagari consonants recognition using MLPNN with five fold cross validation. In: International Conference on Circuits, Power and Computing Technologies. IEEE, Nagercoil, India, pp1222-1226, 2013.

31. Glazko GV and Emmert-Streib F: Unite and conquer: Univariate and multivariate approaches for finding differentially expressed gene sets. Bioinformatics 25: 2348-2354, 2009.

32. Khatri P, Sirota M and Butte AJ: Ten years of pathway analysis: Current approaches and outstanding challenges. PLOS Comput Biol 8: e1002375, 2012.

33. Donato M, Xu Z, Tomoiaga A, Granneman JG, Mackenzie RG Bao R, Than NG, Westfall PH, Romero R and Draghici S: Analysis and correction of crosstalk effects in pathway analysis Genome Res 23: 1885-1893, 2013

34. Bradley EW, Ruan MM, Vrable A and Oursler MJ: Pathway crosstalk between Ras/Raf and PI3K in promotion of M-CSFinduced MEK/ERK-mediated osteoclast survival. J Cell Biochem 104: 1439-1451, 2008.

35. Liu Z-P, Wang Y,Zhang X-S and Chen L: Network-based analysis of complex diseases. IET Systems Biology 6: 22-33, 2012.

36. Barabási AL and Oltvai ZN: Network biology: Understanding the cell's functional organization. Nat Rev Genet 5: 101-113, 2004.

37. Tong AHY, Lesage G, Bader GD, Ding H, Xu H, Xin X, Young J, Berriz GF, Brost RL, Chang M, et al: Global mapping of the yeast genetic interaction network. Science 303: 808-813, 2004.
38. Kelley R and Ideker T: Systematic interpretation of genetic interactions using protein networks. Nat Biotechnol 23: 561-566, 2005.

39. Nasirudeen AM, Wong HH, Thien P, Xu S, Lam KP and Liu DX: RIG-I, MDA5 and TLR3 synergistically play an important role in restriction of dengue virus infection. PLoS Negl Trop Dis 5: e926, 2011.

40. Broquet AH, Hirata Y, McAllister CS and Kagnoff MF: RIG-I/ MDA5/MAVS are required to signal a protective IFN response in rotavirus-infected intestinal epithelium. J Immunol 186: 1618-1626, 2011.

41. Chatterjee P, Weaver LE, Chiasson VL, Young KJ and Mitchell BM: Do double-stranded RNA receptors play a role in preeclampsia? Placenta 32: 201-205, 2011.

42. Wang D, Xia D and Dubois RN: The crosstalk of PTGS2 and EGF signaling pathways in colorectal cancer. Cancers (Basel) 3: 3894-3908, 2011. International (CC BY-NC-ND 4.0) License. 